\title{
New insights into phospholipase D and sphingosine kinase activation in Arabidopsis
}

\author{
Carl K.-Y. Ng ${ }^{1 *}$ and Sylvie Coursol ${ }^{2}$ \\ ' School of Biology and Environmental Science, University College Dublin, Dublin, Ireland \\ 2 UMR 0320/UMR 8120 Génétique Végétale, Institut National de la Recherche Agronomique, Gif-surYYvette, France \\ *Correspondence: carl.ng@ucd.ie
}

\section{A commentary on}

Crosstalk between phospholipase D and sphingosine kinase in plant stress signaling by Liang, G., and Wang, X. (2012). Front. Plant Sci. 3:51. doi: 10.3389/fpls.2012.00051

Since the treatise by Thudichum in 1884 where he observed that "the fatty acids dissolve, while a body remains insoluble, which is of an alkaloid nature, and to which, in commemoration of the many enigmas which it presented to the enquirer, I have given the name of Sphingosin," much is known about the cellular and physiological functions of sphingosine and its phosphorylated derivative, sphingosine-1-phosphate (S1P), in mammals. $\mathrm{S} 1 \mathrm{P}$ is produced in mammalian cells by two sphingosine kinase (SPHK) isoenzymes, SPHK1 and SPHK2, which differ in their temporal and spatial distribution (Maceyka et al., 2012). The SPHK/S1P pathway has been shown to regulate diverse physiological processes ranging from cellular proliferation to survival and pathophysiological processes like osteoporosis, diabetes, and cancer. Mammalian SPHKs can be regulated by numerous effectors. For example, the mammalian SPHK1 is activated by its interaction with TRAF2 (TNF receptor-associated factor 2), with SPHK1 acting as a co-factor for the E3 ligase activity of TRAF2 (Alvarez et al., 2010). The mammalian SPHK2 is part of the repressor complex with histone $\mathrm{H} 3$ and histone deactylases (HADCs) in the nucleus, and the resulting nuclear production of S1P by SPHK2 regulates histone acetylation and transcription (Hait et al., 2009).

In plants, work from the Wang laboratory showed that the two tonoplastic SPHK1 and SPHK2 isoenzymes from Arabidopsis thaliana can be activated by phospholipase $\mathrm{D} \alpha 1$ (PLD $\alpha 1)$-derived phosphatidic acid (PA) species, and that SPHK-derived phytosphingosine-1-phosphate (phyto-S1P) acts upstream of PLD $\alpha 1$ (see this review by Guo and Wang). Guo and Wang have provided a comprehensive review of the crosstalk between the sphingolipid and phospholipid pathways and their potential roles in regulating signaling processes. However, two questions remain: (1) how do PAs activate SPHKs, and (2) how does phyto-S1P activate PLD?

Firstly, work in the mid 1990s by Olivera et al. may provide some clues to the underlying mechanism(s). They showed that mammalian SPHKs can be activated by phosphatidylserine, and to a lesser extent by lysophosphatidylserine, phosphatidylinositol, phosphatidylinositol bisphosphate, cardiolipin, and PA. These SPHK activators are acidic phospholipids and their ability to activate SPHK activity suggests the potential importance of the negative charges on the phospholipid molecules. This is supported by the observation that diacylglycerol, which is structurally similar to PA but lacking the phosphate group did not activate SPHKs (Olivera et al., 1996). The situation appears to differ in the case of $A$. thaliana SPHK1 and SPHK2 as these enzymes do not appear to interact with other phospholipids such as phosphatidylcholine, phosphatidylethanolamine, phosphatidylglycerol, phosphatidylinositol, phosphatidylserine, lysophosphatidylcholine, and lysophosphatidylethanolamine (Guo et al., 2011). In contrast to mammalian SPHK1 and SPHK2 that are localized mainly in the cytosol and the nucleus, respectively, $A$. thaliana SPHK1 and SPHK2 are primarily associated with the tonoplast (Marion et al., 2008; Guo et al., 2011), and thus it remains to be determined if their activation by PA only may be due to their tonoplastic localization. Given the diversity in phospholipid classes and species in eukaryotic cells, it will be interesting, while at the same time challenging, to determine the effects of particular phospholipids on plant SPHK activities, and Wang and co-workers have initiated work in this regard with respect to different PA species (Guo et al., 2011). Additionally, it can be speculated that PA targets (see this review by Guo and Wang) can phosphorylate SPHKs, leading to their activation as previously shown in mammals (Pitson, 2010). Clearly, solving the SPHK structures will be crucial for understanding the regulatory mechanisms that control these enzymes.

Secondly, work from the Wang laboratory showed that phyto-S1P does not activate PLD $\alpha 1$ directly in vitro (Guo et al., 2012). S1P and phyto-S1P were found to stimulate an intracellular calcium increase ( $\mathrm{Ng}$ et al., 2001; Kim et al., 2007), and calcium is a key factor required for PLD $\alpha 1$ activity (see this review by Guo and Wang). Consequently, phyto-S1P may increase cytoplasmic calcium to promote PLD $\alpha 1$ translocation, resulting in PLD $\alpha 1$ activation. On the other hand, work on two A. thaliana mutants disrupted in the $\mathrm{G} \alpha$ subunit of heterotrimeric $\mathrm{G}$ protein showed that they are incapable of initiating stomatal closure when they are challenged by phyto-S1P, suggesting that $\mathrm{G} \alpha$ functions downstream of SPHK/phyto-S1P (Coursol et al., 2005). Additionally, it was shown that PLD $\alpha 1$ physically interacts with G $\alpha$ (Mishra et al., 2006), and thus phytoS1P may activate G $\alpha$ to stimulate PLD $\alpha 1$. Network inference also predicted a positive regulation of $\mathrm{G} \alpha$ by S1P, which in turn positively regulates PLD/PA (Li et al., 2006). The resulting effect on stomatal closure is likely to be mediated by PA inhibition of the type $2 \mathrm{C}$ protein phosphatase $\mathrm{ABI} 1$, a negative regulator of ABA signaling (Li et al., 2006). It will be interesting to see if as predicted by $\mathrm{Li}$ et al. (2006), ABA inhibition of ABI1 phosphatase activity is impaired in sphk mutants unable to produce phyto-S1P.

We envisage that determination of the structure-activity relationships between phospholipids and SPHKs will yield interesting clues as to the underlying mechanisms for phospholipid activation of 
SPHKs. Additionally, it will be interesting to see if non-seed land plants and monocots show similar trends and responses as sphingosine is present in these plants (Islam et al., 2012), and may have an effect on which long chain bases are used in the SPHK and PLD-mediated pathways.

\section{ACKNOWLEDGMENTS}

Research in the authors' laboratories are supported by funds from Science Foundation Ireland (SFI) Research Frontiers Programme and Equipment Grants (06/SFI/ RFP/GEN034, 06/SFI/RFP/GEN034ES, 08/ SFI/RFP/EOB1087) to Carl K.-Y.Ng, and by the long-term public-private AMAIZING research initiative to Sylvie Coursol.

\section{REFERENCES}

Alvarez, S. E., Harikumar, K. B., Hait, N. C., Allegood, J., Strub, G. M., Kim, E. Y., Maceyka, M., Jiang, H., Luo, C., Kordula, T., Milstien, S., and Spiegel, S. (2010). Sphingosine-1-phosphate is a missing cofactor for the E3 ubiquitin ligase TRAF2. Nature 465, 1084-1088.

Coursol, S., Le Stunff, H., Lynch, D. V., Gilroy, S., Assmann, S. M., and Spiegel, S. (2005). Arabidopsis sphingosine kinase and the effects of phytosphingo- sine-1-phosphate on stomatal aperture. Plant Physiol. $137,724-737$.

Guo, L., Mishra, G., Markham, J. E., Li, M., Tawfall, A., Welti, R., and Wang, X. (2012). Connections between sphingosine kinase and phospholipase $\mathrm{D}$ in the abscisic acid signaling pathway in Arabidopsis. J. Biol. Chem. doi: 10.1074/jbc.M111.274274

Guo, L., Mishra, G., Taylor, K., and Wang, X. (2011). Phosphatidic acid binds and stimulates Arabidopsis sphingosine kinases. J. Biol. Chem. 286, 13336-13345.

Hait, N. C., Allegood, J., Maceyka, M., Strub, G. M., Harikumar, K. B., Singh, S. K., Luo, C., Marmorstein, R., Kordula, T, Milstien, S., and Spiegel, S. (2009). Regulation of histone acetylation in the nucleus by sphingpsine-1-phosphate. Science 325, 1254-1257.

Islam, M. N., Jacquemot, M. P., Coursol, S., and Ng, C. K. (2012). Sphingosine in plants-more riddles from the Sphinx? New Phytol. 193, 51-57.

Kim, M. K., Park, K. S., Lee, H., Kim, Y. D., Yun, J., and Bae, Y. S. (2007). Phytosphingosine-1-phosphate stimulates chemotactic migration of L2071 mouse fibroblasts via pertussis toxin-sensitive G-proteins. Exp. Mol. Med. 39, 185-194.

Li, S., Assmann, S. M., and Albert, R. (2006). Predicting essential components of signal transduction networks: a dynamic model of guard cell abscisic acid signaling. PLoS Biol. 4, e312. doi: 10.1371/journal.pbio.0040312

Maceyka, M., Harikumar, K. B., Milstein, S., and Spiegel, S. (2012). Sphingosine-1-phosphate signaling and its role in disease. Trends Cell Biol. 22, 50-60.

Marion, J., Bach, L., Bellec, Y., Meyer, C., Gissot, L., and Faure, J. D. (2008). Systematic analysis of protein subcellular localization and interaction using highthroughput transient transformation of Arabidopsis seedlings. Plant J. 56, 169-179.

Mishra, G., Zhang, W., Deng, F., Zhao, J., and Wang, X. (2006). A bifurcating pathway directs abscisic acid effects on stomatal closure and opening in Arabidopsis. Science 312, 264-266.

Ng, C. K., Carr, K., McAinsh, M. R., Powell, B., and Hetherington, A. M. (2001). Drought-induced guard cell signal transduction involves sphingosine-1-phosphate. Nature 410, 596-599.

Olivera, A., Rosenthal, J., and Spiegel, S. (1996). Effect of acidic phospholipids on sphingosine kinase. J. Cell. Biochem. 60, 529-537.

Pitson, S. M. (2010). Regulation of sphingosine kinase and sphingolipid signaling. Trends Biochem. Sci. 36, 97-107.

Received: 09 March 2012; accepted: 09 March 2012; published online: 27 March 2012.

Citation: Ng CK-Y and Coursol S (2012) New insights into phospholipase D and sphingosine kinase activation in Arabidopsis. Front. Physio. 3:67. doi: 10.3389/ fphys.2012.00067

This article was submitted to Frontiers in Plant Physiology, a specialty of Frontiers in Physiology.

Copyright (๑ $2012 \mathrm{Ng}$ and Coursol. This is an openaccess article distributed under the terms of the Creative Commons Attribution Non Commercial License, which permits non-commercial use, distribution, and reproduction in other forums, provided the original authors and source are credited. 